\title{
Peripartum pulmonary embolism: Anesthetic and surgical considerations
}

\author{
Nissar Shaikh ${ }^{1 *}$, Firdous Ummunnisa ${ }^{2}$, Naseera Aboobacker ${ }^{2}$, Mustafa Gazali $^{1}$, Ousama Kokash ${ }^{1}$ \\ ${ }^{1}$ Department of Anesthesia, Hamad Medical Corporation, Weil Cornell Medical College, Doha, Qatar \\ ${ }^{2}$ Department of Obstetrics and Gynecology, Hamad Medical Corporation, Doha, Qatar \\ Email: ${ }^{*}$ nissatfirdous99@gmail.com
}

Received 1 November 2012; revised 3 December 2012; accepted 11 December 2012

\begin{abstract}
Pregnancy and peripartum period leads to Virchow's triad (hypercoagulability, venous stasis and vascular injury) thereby increasing the risk of thromboembolism by many folds in these patients. Accurate diagnosis of peripartum pulmonary embolism is pertinent for reducing morbidity and mortality. Accurate diagnosis is also vital for avoiding the adverse effects of unwanted anticoagulation in pregnant mother and fetus in a patient wrongly diagnosed with this condition. Computerized tomographic pulmonary angiography has a high specificity and sensitivity in comparison to ventilation/perfusion scan for diagnosis of peripartum pulmonary embolism (PPE). It has a lower fetal radiation exposure and aids in arriving at an alternative diagnosis, if PPE is absent. Low molecular weight heparin is the medication of choice in the treatment of peripartum pulmonary embolism. Thrombolysis is considered in patients with massive PPE and hemodynamic instability, refractory hypoxia or right ventricular dysfunction. Regional anesthesia/analgesia can be given safely in these patients. We report two cases of $\mathrm{PPE}$ and review the anesthetic and surgical consideration.
\end{abstract}

Keywords: Peripartum Pulmonary Embolism; Computerized Tomographic Pulmonary Angiography; Low Molecular Weight Heparin

\section{INTRODUCTION}

Either congenital or acquired thrombophilia's are the most important risk factor for the development of peripartum pulmonary embolism (PPE). During pregnancy the pro-coagulant factors, fibrinogen, factor $\mathrm{v}$, vii, ix and $\mathrm{x}$ leading to the enhanced thrombin generation, at the same time activity of protein $\mathrm{S}$ is decreased and activated protein $\mathrm{C}$ resistance is increased. This overall situation

\footnotetext{
*Corresponding author.
}

results in a hypercoagulable state [1].

The rate of pulmonary embolism (PE) in the peripartum period is fivefold higher in comparison to the same aged non-pregnant female [2]. This increased risk of peripartum pulmonary embolism (PPE) is a combined effect of hypercoagulability, venous stasis and vascular injury. Peripartum pulmonary embolism (PPE) is one of the leading causes of maternal morbidity and mortality in developed countries. Asian pregnant women have a significant lower risk of venous thromboembolism compared to white pregnant women (1.07 vs 1.75/1000 deliveries) [3]. Pulmonary thromboembolism in pregnant Japanese population is comparatively rare; but in last decade incidence increased by 6.5 folds [4]. No literature is available about peripartum pulmonary embolism in India but overall incidence of pulmonary embolism is lower in Indian population [5]. In Saudi population pulmonary embolism is the second leading cause of maternal mortality [6]. It is of vital importance to diagnose PPE, to avoid the higher morbidity and mortality of under-diagnosis; at the same time to exclude the over diagnosis and to avoid unnecessary adverse effects of anticoagulation to mother and the fetus [2].

Pregnancy and peripartum period lead to Virchow's triad (hypercoagulability, venous stasis and vascular injury) and thus increasing the risk of thromboembolism by many folds in these patients. Developed countries successfully controlled the peripartum morbidity and mortality, from hemorrhage and sepsis; but the pulmonary embolism remained the leading cause of maternal death [1]. Pulmonary embolism is either under diagnosed by attributing the sign and symptoms of pregnancy or it is over diagnosed in pregnant patient with chest pain, respiratory distress and collapse. Proper diagnosis of peripartum pulmonary embolism is essential for reducing morbidity and mortality of pregnant mother as well as the fetus. Hence it is worth to diagnose peripartum pulmonary embolism (PPE) properly and manage accordingly. We report two cases of PPE and review the anesthetic and surgical consideration. 


\subsection{Case 1}

A 39-year gravida 5, para 4 with gestational diabetes mellitus, underwent elective lower segment cesarean section (LSCS), during LSCS had bouts of desaturation which improved by adding physiological positive endexpiratory pressure (PEEP). In recovery room had respiratory distress and desaturated to $80 \%$, needed endotracheal intubation and ventilation. Patient was shifted to the surgical intensive care unit (SICU) for further management. In SICU patients remained haemodynaemically stable, chest x-ray showed right basal segmental collapse, she was started on aggressive chest physiotherapy sedated with propofol and remifentanil analgesia. On day 2: she had convulsions; magnesium sulphate was added to the therapy. Computerized tomography (CT) of head was normal but CT chest showed right pulmonary artery thrombus (Table 1): she was started on therapeutic dose enoxaparin sodium; Weaned from ventilator and extubated on day 3. She was awake and haemodynaemicaly stable, transferred to the ward on day 4 from there discharged home on enoxaparin therapy to be followed in out-patient clinics.

\subsection{Case 2}

A 22-year female primigravida in 34 weeks of gestation, known case of systemic lupus erythematous and antiphospholipid antibodies syndrome presented to emergency department with shortness of breath, tachypnea and tachycardia but stable blood pressure.

Echocardiogram showed Left pulmonary artery thrombosis pulmonary embolism with severe pulmonary hypertension (right ventricular systolic pressure of $85 \mathrm{~mm}$ of $\mathrm{Hg}$ ) and severe right ventricular dysfunction, started her on enoxaparin sodium (Table 1) and digoxin. Next day underwent LSCS, postoperatively shifted to SICU. She was weaned and extubated on day 2. She remained haemodynaemicaly stable. Transferred to the ward and

Table 1. Summary of diagnosis and management of our two cases.

\begin{tabular}{ccc}
\hline & Case 1 & Case 2 \\
\hline Pregnancy & Multipara & Primigravida \\
$\begin{array}{c}\text { Timing of Pulmonary } \\
\text { Embolism }\end{array}$ & Post-Partum & Ante-Partum \\
Risk Factors & $\begin{array}{c}\text { Gestational Diabetes } \\
\text { Mellitus/Eclampsia }\end{array}$ & Thrombophilia \\
Respiratory Distress & Yes & Yes \\
Imaging Study & Tomographic Angiogram & $\begin{array}{c}\text { Echocardiogram } \\
\text { Therapy }\end{array}$ \\
& Low Molecular & Low Molecular \\
& Heparin & Heparin \\
\hline
\end{tabular}

then discharged home to be followed in out-patient department.

\section{EPIDEMIOLOGY}

The incidence of PE in pregnancy and peripartum period varies between 1 to 3 per 3000 deliveries [7]. During each trimester of pregnancy the risk of PE is same but it will increase by 20-fold in the postpartum period [7]. Du- ring pregnancy and Peripartum period. Eighty percent of thromboembolism is venous [7]. Eighty percent of throm- boembolism is consequent to deep venous thrombosis (DVT) and only 20\% presents as pulmonary artery throm- bosis.

\section{ETIOPATHOLOGY}

In pregnancy DVT is more common in left lower limb, due to compression of the left common iliac vein in-between lumbar vertebral body and the right common iliac artery [7]. There are various risk factors, which will increase the risk of thrombosis during pregnancy and postpartum period. 1) Age, the incidence of PE is double after 35 years [8]; 2) Parity: the risk of PE increases with the increase in parity, particularly the risk is more after three pregnancies [8]; 3) obesity [8,9]; 4) operative delivery: up to 8 folds depending on emergency or elective procedure [9]; 5) Estrogen administration; 6) Pregnancy with heart disease, sickle cell disease, lupus, diabetes, hypertension and smoking; 7) Pregnancy complicated with multiple gestation, hyperemesis, peripartum hemorrhage, massive transfusion and the postpartum infection; 8) Thrombophilia is present in up to 50\% of the women who had venous thromboembolism during pregnancy or in peripartum period [10]; 9) History of thrombosis is an important risk factor, recurrent episodes account for $15 \%$ to $25 \%$ of all thromboembolic events during pregnancy or peripartum period.

Venodilatation and veno-stasis during pregnancy begins in first trimester and reaches to the maximum at 36 weeks of gestation, addition to it compression of pelvic veins by gravid uterus and compression of left iliac vein by the artery increases the risk for thromboembolism [1, $11]$.

Physiological changes of pregnancy causes increase in procoagulant factors and decease in natural anticoagulants leading to a Hypercoagulable state [1,11]. Vascular and endothelial injury occurring during normal and surgical delivery will increase the risk of PE.

\section{CLINICAL FEATURES \& DIAGNOSIS}

The important therapeutic aspect of peripartum pulmonary embolism (PPE) is the proper diagnosis. The clinical manifestations of PE are not different in pregnancy or 
Peripartum period, but breathlessness is a common feature in pregnancy, if it is absent at presentation, we can exclude PE in up to $90 \%$ of patients [12]. Arterial blood gas (ABG) in sitting position and electrocardiogram should be done. The D-Dimer levels are non-specific for diagnosis of PE during pregnancy and postpartum period, as levels are raised during pregnancy and return to normal six weeks postpartum [13]. Echocardiography will be another non-invasive and radiation free tool for the diagnosis of major pulmonary embolism. It will show typical "McConnell sign” which is right ventricular (RV) free wall hypokinesia in the presence of normal RV apical contractility with signs of RV pressure load. Echocardiography will also rule-out other cardiac etiologies for the respiratory distress [14].

Fear of fetal radiation toxicity should not deprive mother of timely and proper diagnostic evaluation. Animal data revealed that radiation exposure of 0.1 gray (Gy) at any time during gestation is considered as threshold above which will lead to fetal congenital abnormalities [15]. Radiation exposure from the single $\mathrm{x}$-ray chest is equal to the extra radiation exposure during a trans-Atlantic flight. We can also cover the abdomen with a lead sheath to prevent fetal radiation exposure [14]. Ventilation/perfusion (V/Q) imaging is the most commonly performed test for the diagnosis of PE during pregnancy. The radiation dose can be reduced by doing half dose perfusion and proceed for ventilation scan. Majority of pregnant patients will have normal V/Q scan and interesting that normal V/Q scan will not rule-out the PE; only 5\% will have a high probability scan $[14,15]$.

Computerized tomographic pulmonary angiography (CTPA) is a well validated test for PE with sensitivity and specificity of $94 \%$ and $100 \%$ respectively [16]. The advantages of CTPA over the V/Q scan are direct visualization of thrombus and if no embolism is detected it gives the alternative diagnosis such as pneumonia, pulmonary edema or massive pleural effusion. When compared for fetal radiation exposure V/Q scan has 3 times (130 Versus 370 uGy) more exposure than CTPA. [17] All types of radiation carries risk to the fetus, but this risk should be weighed against the benefits of diagnosis and treatment of maternal PE. When considering the radiation risk to the fetus, we shouldn't forget the radiation exposure to the maternal breast. When compared V/Q scan and CTPA, the breast radiation exposure is more with later technique. The imaging techniques to diagnose $\mathrm{PE}$ in pregnant patient (Figure 1) are summarized in the form of algorithm. Various radiological techniques and their advantages are described in Table 2. Doppler ultrasound has advantage of diagnosing DVT without exposure to the ionizing radiation; CTPA has a high specificity and sensitivity in comparison to V/Q scan for diagnosis of PE [18]. Pulmonary embolism during preg- nancy must be differentiated from amniotic fluid embolus and chorionic carcinoma. Chorionic carcinoma causes similar picture as PE, due to the tumor cells in the pulmonary circulation. Amniotic fluid embolus can be differentiated by prolonged coagulation parameters.

\section{MANAGEMENT}

Thrombolysis is considered in patients with massive PPE and hemodynamic instability, refractory hypoxia or right ventricular dysfunction (Figure 2).

Heparin is the most commonly used anticoagulant in the treatment of PPE; other anticoagulants used are warfarin, factor $\mathrm{x}$ inhibitors, heparinoid derivatives and direct thrombin inhibitors. If the CTPA is negative, there is no need for the anticoagulation.

Heparin doesn't cross the placenta and not secreted in the breast milk, hence it is the drug of choice for treatment of PE during pregnancy. Heparin infusion can be stopped few hours before delivery or surgery or its effect can be reversed by the protamine sulphate. LMWH has advantages of convenient dosage, no need of coagulation monitoring and less side effects.

Warfarin is a vitamin $\mathrm{K}$ antagonist, it crosses placenta, its use is teratogenic, causes miscarriage and it is associated with major fetal central nervous system abnormalities, and full anticoagulation can cause fetal retroperitoneal and intracerebral hemorrhages [19]. Selective factor $\mathrm{x}$ inhibitors and direct thrombin inhibitors are used when $\mathrm{PE}$ is associated with heparin induced thrombocytopenia.

\subsection{Peripartum Thromboprophylaxis}

One has to be very careful in treating PE in the peripartum period particularly at 37 weeks of gestation or beyond, as the mortality is high with untreated PE at the same time high chances of bleeding through genital tract due to anticoagulation therapy [20]. If PE is diagnosed near the term, induction of labor and anticoagulation with UFH is advised, as action of UFH is predictable and it can be reversed (Figure 2), UFH can be stopped once

Table 2. Risk and benefits of imaging studies in PPE.

\begin{tabular}{ccc}
\hline Imaging Study & Benefit & Risk \\
\hline $\begin{array}{c}\text { Computerized } \\
\text { Tomographic } \\
\text { Pulmonary } \\
\text { Angiogram (CTPA) }\end{array}$ & $\begin{array}{c}\text { Will show thrombus, } \\
\text { gives alternative } \\
\text { diagnosis and lower } \\
\text { fetal radiations }\end{array}$ & $\begin{array}{c}\text { Higher breast } \\
\text { radiations; use of } \\
\text { contrast }\end{array}$ \\
$\begin{array}{c}\text { Ventilation/Perfusion } \\
\text { Scan (V/Q Scan) }\end{array}$ & $\begin{array}{c}\text { Lower maternal } \\
\text { breast radiation }\end{array}$ & $\begin{array}{c}\text { No clinical } \\
\text { decisions or no } \\
\text { alternative diagnosis }\end{array}$ \\
$\begin{array}{c}\text { Compression } \\
\text { Ultrasonography } \\
\text { (CUS) }\end{array}$ & $\begin{array}{c}\text { No radiations, non } \\
\text { invasive; potential to } \\
\text { detect deep venous } \\
\text { thrombosis }\end{array}$ & Low sensitivity \\
\hline
\end{tabular}




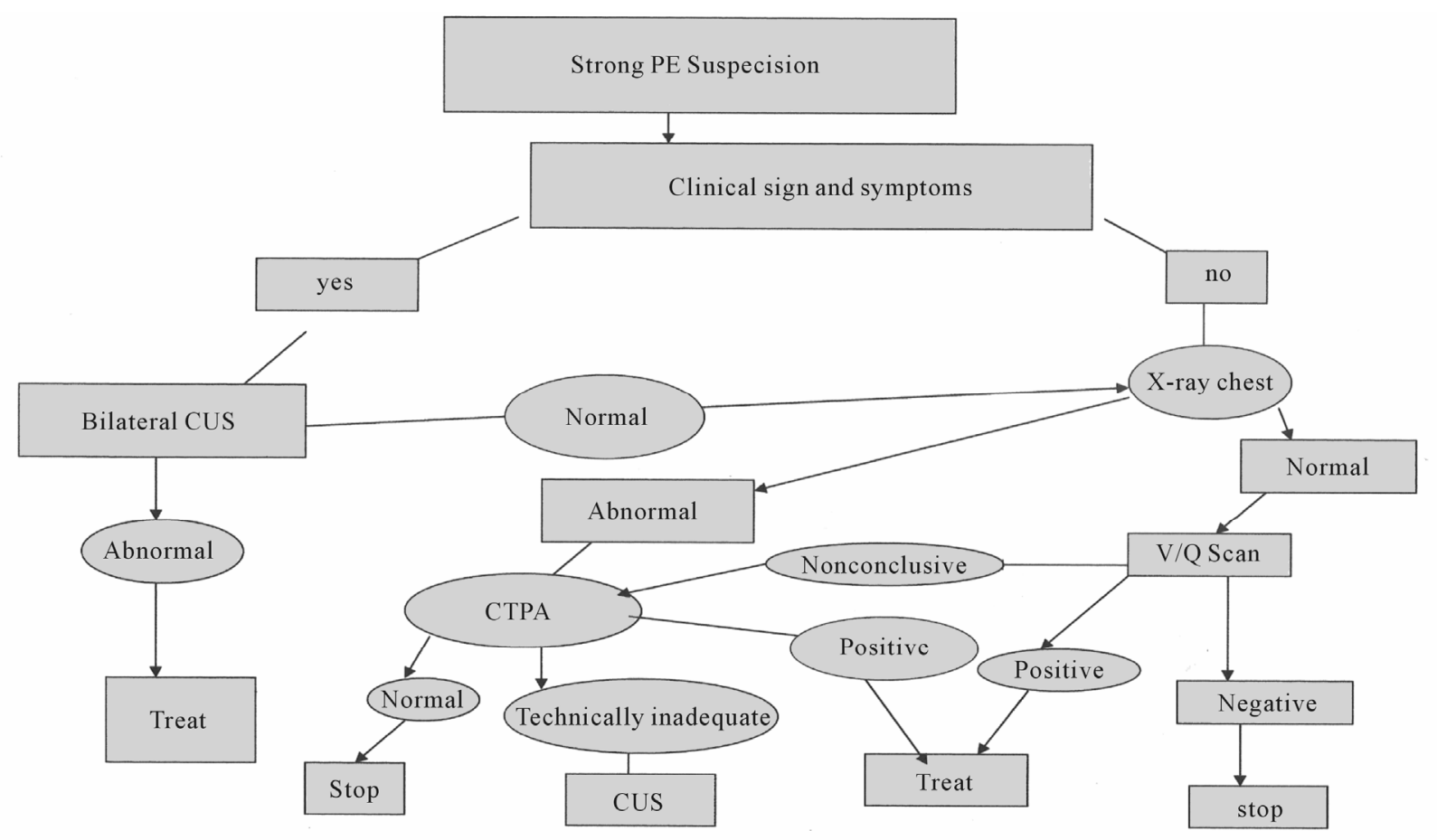

\section{Modified from Leung AN et al. Am J Respir Crit Care Med 2011; 184: 1200-8}

Figure 1. Algorithm for the diagnosis of peripartum pulmonary embolism.

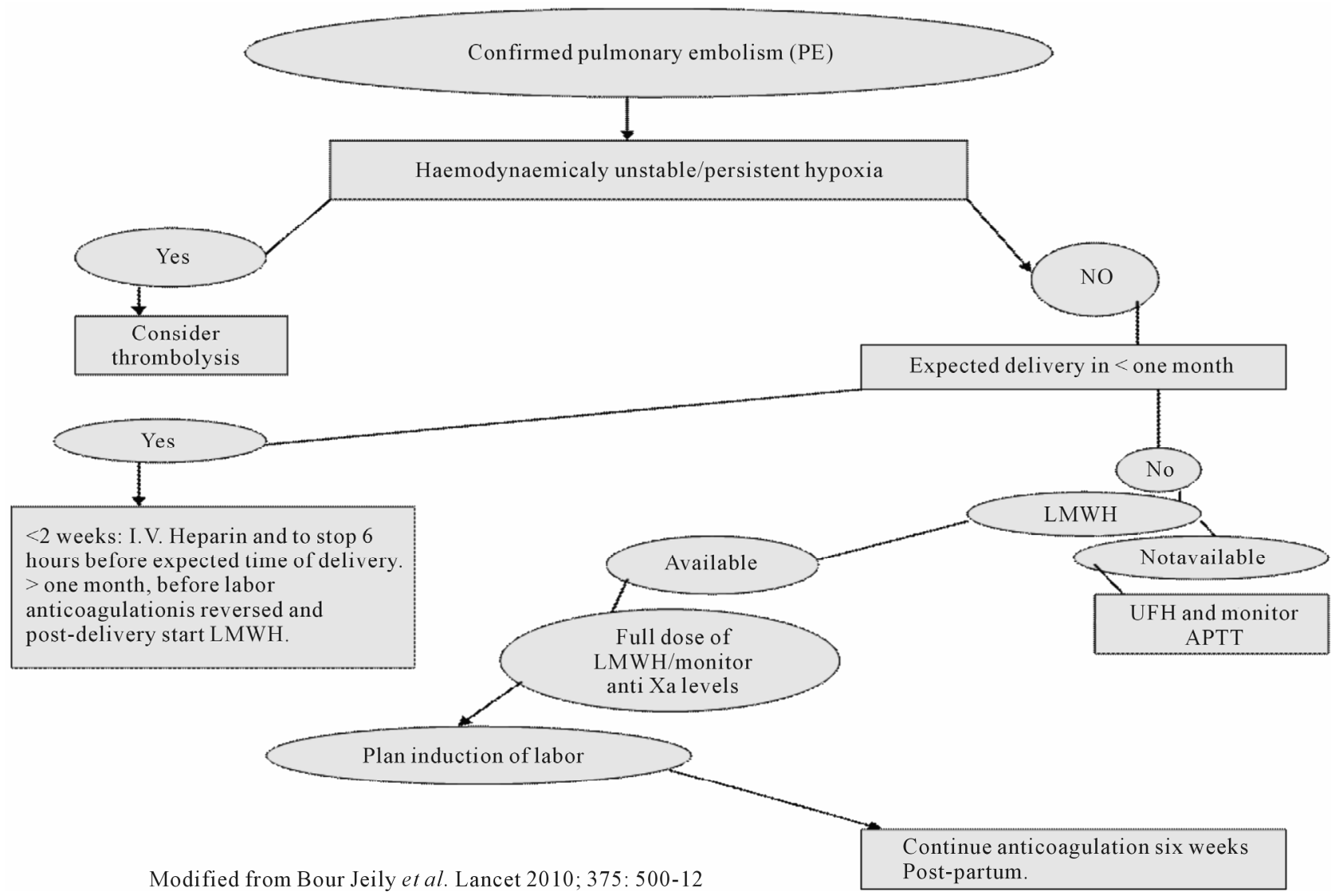

Figure 2. Treatment of peripartum pulmonary embolism. 
patient goes into active labor or easily reversed if delivery occurs; the duration without anticoagulation should be less than 12 hours. Restarting of anticoagulation after delivery should be adjusted according to the risk of reoccurrence of PE and bleeding. In the peripartum period, UFH should be resumed once bleeding stopped.

Duration of post-partum anticoagulation depends on various risk factors for PE. Patients with thrombophilia or anticardiolipin antibodies or recurrent unprovoked DVT should continue anticoagulation for rest of the life. In patients with temporary risk factors for PE (plaster cast, immobilization for more than 72 hours, major surgery or trauma) anticoagulation three months post-partum is enough. But for patients who developed PE without any exacerbating risk factors; anticoagulation should be continued for six months (Figure 3) in post-partum period [21].

\subsection{Anesthetic Management}

Anesthetic management of pregnant patients with pulmonary embolism is rarely described and limited to the case reports only. Patients with fetal distress/right failure and other obstetrical indication for cesarean section; can have general anesthesia.
Many anesthetists are reluctant to use regional anesthesia/analgesia in peripartum patients with pulmonary embolism on anticoagulation therapy, due to the fear of hemorrhagic and neurological complications (spinal/epidural hematoma); there is not enough data regarding efficacy of anticoagulants in pregnancy and not much literature is available about safety and risk of regional anesthesia and analgesia during the peripartum period.

Moen et al. reported the incidence of 1:200,000 neurological complications with regional anesthesia in pregnant patients [22]. But up till now there are no published reports about spinal hematoma in parturient associated with antithrombotic therapy; at the same time there are reports of successful use of regional anesthesia for the pregnant patients with pulmonary embolism on antithrombotic medications under going surgical delivery [23].

Following regional anesthesia/analgesia spinal/epidural hematoma is more likely to occur in patients on anticoagulant therapy. It is less likely with a single-shot spinal procedure, as the spinal needle is smaller in size and no indwelling catheters; leading to lesser trauma. Pregnant patients with pulmonary embolism on anticoagulation therapy have an increased risk of hemorrhage in the

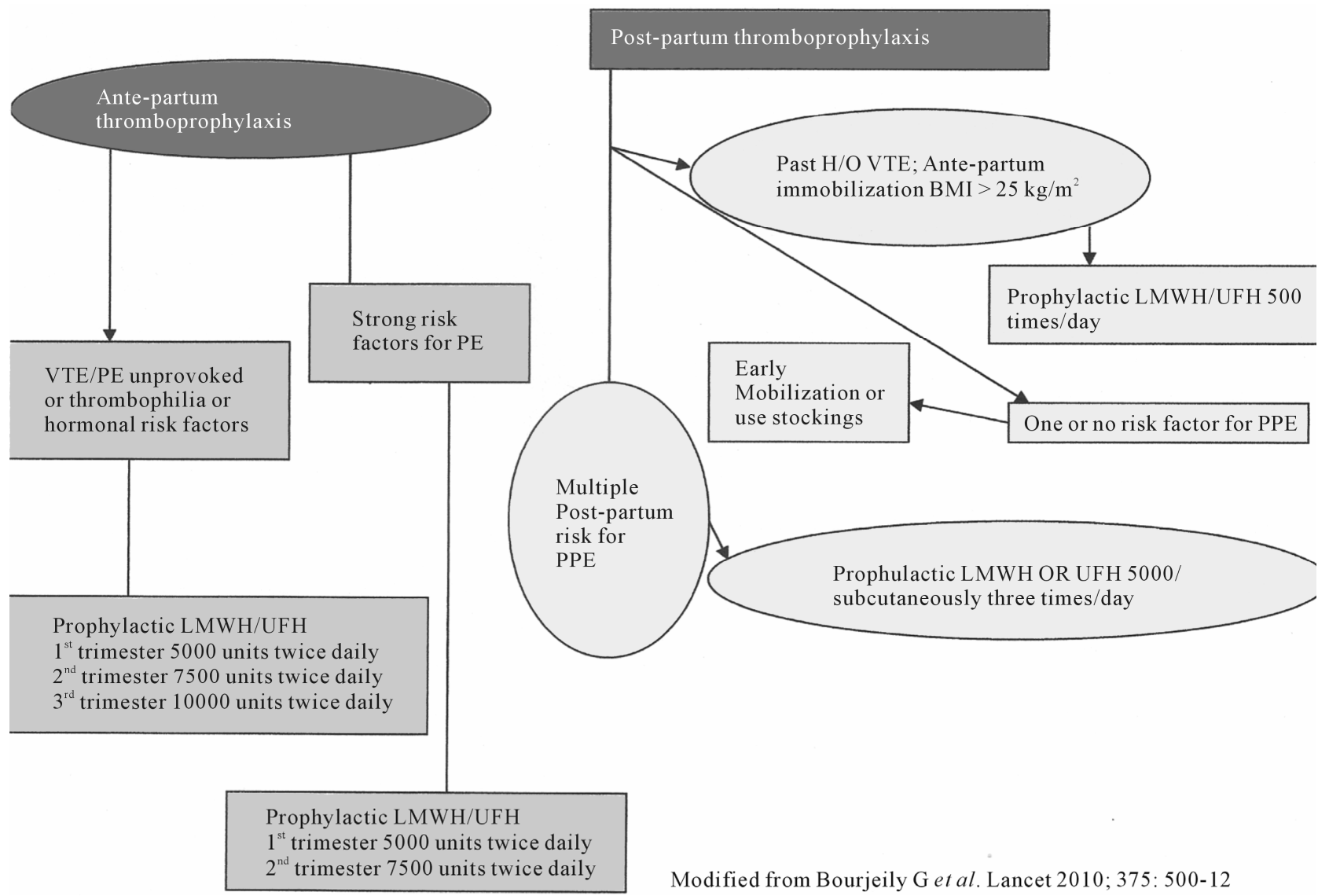

Figure 3. Peripartum therapy for pulmonary embolism. 
neuroaxis, following regional anesthesia; hence one has to consider what type of anticoagulant patient is receiving, dosage and time of anticoagulant administration. These patients's coagulation status should be meticulously monitored.

If patient is receiving unfarctionated heparin (UFH), we should monitor activated partial thromboplastin time (APTT) and platelet count (as these patients can develop heparin induced thrombocytopenia). If patients are on LMWH, the routine coagulation test is not useful and we should monitor factor $\mathrm{x}$ activity. Patients on warfarin, should be followed with prothrombin time (PT) and international normalized ratio (INR).

As per the American society of regional anesthesia and pain medicine, following recommendations are made for regional anesthesia/analgesia [24].

1) Use of aspirin and other nonsteriodal anti-inflammatory medications should not influence the decision to place a neuroaxial block, but it should be avoided if patient receiving these medications in combination with UFH, LMWH or vitamin K inhibitors.

2) Based on available reports of spinal hematomas, it is recommended that a waiting period of 14 days between the last dose of ticopidine; 7 days after the last dose of clopidogrel and placement of neuroaxial block. For patients receiving glycoprotein IIb/IIIa receptor antagonist, neuroaxial techniques should be avoided for 24 to 48 hours.

3) Women taking vitamin $\mathrm{K}$ antagonist should be shifted to intravenous or subcutaneous UFH/LMWH no later than 36 weeks of gestation.

4) Women on LMWH should be transitioned to intravenous or subcutaneous UFH no later than 36 hours before induction of labor or cesarean delivery.

5) Intravenous heparin should be discontinued four to six hours before scheduled delivery.

6) Patients on subcutaneous UFH prophylaxis 5000 units twice daily, neuroaxial techniques are not contraindicated. However for the safety of neuroaxial techniques patient receiving three times daily dosage of greater than 10,000 units daily or if the dose of UFH is not known; it is advisable to confirm that activated partial thromboplastin time has returned to normal prior to performing the procedure.

7) Post partum prophylactic anticoagulation with heparin (UFH/LMWH) should be delayed 12 hours after vaginal delivery and 24 hours after cesarean section [24].

Pregnant patients on UFH therapy, who recently had epidural catheterization, heparin should not be given for one hour after insertion. UFH should be hold 4 - 6 hours before procedure and resumed 4 - 6 hours after surgical procedure. If patient is receiving $\mathrm{LMWH}$; the morning dose should be hold on the day of procedure. Epidural catheter should be removed after 2 to 4 hours of UFH hold or 2 hours before the post procedure dose of LMWH [25].

\section{CONCLUSION}

In developed countries peripartum pulmonary embolism is the leading cause of maternal mortality. Pulmonary embolism is either under diagnosed by attributing the sign and symptoms to the changes of pregnancy or over diagnosed in pregnant patient with chest pain, respiratory distress and collapse. It is important to diagnose pulmonary embolism accurately during pregnancy and peripartum period to avoid higher morbidity and mortality. The main risk factor for peripartum pulmonary embolism is congenital or acquired thrombophilia. During pregnancy the procoagulant factors, fibrinogen, factor $\mathrm{v}$, vii, ix and $\mathrm{x}$ leads to the enhanced thrombin generation, at the same time the activity of protein $\mathrm{s}$ is decreased and activated protein c resistance is increased. This overall situation results in a hypercoagulable state. CTPA has a high specificity and sensitivity in comparison to V/Q scan for diagnosis of PE. It has a lower fetal radiation exposure and gives alternative diagnosis, if PE is absent. LMWH are the medication of choice, as they are as effective as unfractionated heparin (UFH) and their long term use is as effective as vitamin $\mathrm{k}$ antagonist. If PE is diagnosed near the term, induction of labor and anticoagulation with UFH is advised, as the action of UFH is predictable and can be reversed, UFH can be stopped once patient goes into active labor or easily reversed if delivery occurs, the duration without anticoagulation should be less than 12 hours. Regional anesthesia/analgesia can be given safely in these patients with strict follow up of the guidelines for holding and reinitiating the antithrombotic medications. If patient is known to have thrombophilia, previous unprovoked PE/DVT during pregnancy; obese patients should receive thromboprophylaxis.

\section{REFERENCES}

[1] Bourjeily, G., Paidas, M., khalil, H., Rosene-Montella, K. and Rodger, M. (2010) Pulmonary embolism in pregnancy. Lancet, 375, 500-512. doi:10.1016/S0140-6736(09)60996-X

[2] Mathew, S. (2006) Imaging pulmonary embolism in pregnancy: What is the most appropriate imaging protocol? British Journal of Radiology, 79, 441-444. doi:10.1259/bjr/15144573

[3] James, A.H., Jamison, M.G., Brancazio, L.R., et al. (2006) Venous thromboembolism during pregnancy and the post-partum period. Incidence, risk factors and mortality. American Journal of Obstetrics \& Gynecology, 194, 1311-1315. doi:10.1016/j.ajog.2005.11.008

[4] Kobayashi, T., Nakabayashi, M., Ishikawa, M., Adachi, T., kobashi, G., Maeda, M. and Ikenoue, T. (2008) Pul- 
monary thromboembolism in obstetrics and gynecology increased by 6.5 folds over past decade in Japan. Circulation Journal, 72, 753-756. doi:10.1253/circj.72.753

[5] Khosla, R. (2006) Diagnosis of pulmonary embolism. Indian Journal of Critical Care Medicine, 10, 105-111.

[6] Al-Gahtani, F.H. (2009) Pregnancy associated venous thromboembolism. Part 1-Deep venous thrombosis. Saudi Medical Journal, 1, 13-23.

[7] Heit, J.A., Kobberuig, C.E., James, A.H., et al. (2005) Trends in the incidence of venous thromboembolism during pregnancy or post-partum: 30 years of population based study. Annals of Internal Medicine, 143, 697-706.

[8] Macklon, N.S. and Greer, I.A. (1996) Venous thromboembolic disease in obstetric and gynecology: The Scottish experiences. Scottish Medical Journal, 41, 83-86.

[9] James, A.H., Jamison, M.G., Brancazio, L.R., et al. (2006) Venous thromboembolism during pregnancy and postpartum period: Incidence, risk factors and mortality. American Journal of Obstetrics \& Gynecology, 194, 1311-1315. doi:10.1016/j.ajog.2005.11.008

[10] Robertson, L., Wu, O., Langhorne, P., et al. (2006) Thrombophilia in pregnancy: A systematic review. British Journal of Haematology, 132, 171-196. doi:10.1111/j.1365-2141.2005.05847.x

[11] Rosenkranz, A., Hiden, M., Leschnik, B., Weiss, E.C., Schlembach, D., Lang, U., et al. (2008) Calibrated automatic thrombin generation in normal uncomplicated pregnancy. Thrombosis and Haemostasis, 99, 331-337.

[12] Leung, A.N., Bull, T.M., Jaeschke, R., Lochhood, C.J., Boiselle, P.M., Hurwitz, L.M., et al. (2011) An official American thoracic society/society of thoracic radiology clinical practice guigline: Evaluation of suspected pulmonary embolism in pregnancy. American Journal of Respiratory and Critical Care Medicine, 184, 1200-1208. doi:10.1164/rccm.201108-1575ST

[13] Morse, M. (2004) Establishing a normal range for DDimer levels through pregnancy to aid in diagnosis of pulmonary embolism and deep venous thrombosis. Journal of Thrombosis and Haemostasis, 2, 1202-1204. doi:10.1111/j.1538-7836.2004.00776.x

[14] de Swlet, M. (1999) Management of pulmonary embolus in pregnancy. European Heart Journal, 20, 1378-1385. doi:10.1053/euhj.1999.1584

[15] Ginsberg, J.S., Hirsh, J., Rainbow, A.J. and Coates, G. (1989) Risk to the fetus of radiological procedures used in the diagnosis of maternal venous thromboembolism disease. Journal of Thrombosis and Haemostasis, 61, 189-196.
[16] Blachere, H., Latrabe, V., Montaudon, M., Valli, N., Couffinhal, T., Raherisson, et al. (2000) Pulmonary embolism revealed on helical CT angiography: Comparison with ventilation/perfusion radionuclide lung scanning. American Journal of Roentgenology, 174, 1041-1047.

[17] Winer-Muram, H.T., Boone, J.M., Brown, H.L., Jennings, S.G., Mabie, W.C. and Lombard, G.T. (2000) Pulmonary embolism in pregnant patients; fetal radiation dose with helical CT. Radiology, 224, 487-492. doi:10.1148/radiol.2242011581

[18] Powell, T. and Muller, N.L. (2003) Imaging of acute pulmonary thromboembolism: Should spiral computed tomography replace the ventilation perfusion scan? Clinics in Chest Medicine, 24, 29-38. doi:10.1016/S0272-5231(02)00074-6

[19] Hall, J.G., Pauli, R.M. and Wilson, K.M. (1980) Maternal and fetal sequelae of anticoagulation during pregnancy. American Journal of Medicine, 68, 122-140. doi:10.1016/0002-9343(80)90181-3

[20] Kearon, C. and Hiresh, J. (1997) Management of anticoagulation before and after surgery. The New England Journal of Medicine, 336, 1566-1511. doi:10.1056/NEJM199705223362107

[21] Bates, S.M., Greer, I.A., Pabinger, I., Sofaer, S. and Hirsh, J. (2008) Embolism, thrombophilia, antithrombotic therapy and pregnancy. American College of chest physician evidence based clinical guidelines. Chest, 56, 844-886. doi:10.1378/chest.08-0761

[22] Moen, V., Dahlgren, N. and Irestedt, L. (2004) Severe neurological complications after central neuroaxial blockades in Sweden 1990-1999. Anesthesiology, 101, 950-959. doi:10.1097/00000542-200410000-00021

[23] Kitao, T., Veno, Y., Sekikawa, A., Tatsumi, Y., Nakashima, M., Kan, K., et al. (2008) Anesthetic management in a patient with pulmonary embolism. Masuli, 57, 761763.

[24] Horlocker, T.T., Wedel, D.J., Rowlingson, J.C., et al. (2010) Regional anesthesia in patients receiving antithrombotic or thrombolytic therapy. American society of regional anesthesia and pain management, evidence based guidelines. Regional Anesthesia and Pain Medicine, 35, 64. doi:10.1097/AAP.0b013e3181c15c70

[25] Horlocker, T.T., Wedel, D.J., Roswlingson, J.C., Enneking, K., Kopp, S.L., Benzon, H.T., et al. (2012) Regional anesthesia in patient receiving antithrombotic or thrombolytic therapy. Regional Anesthesia and Pain Medicine, 35, 64-74. doi:10.1097/AAP.0b013e3181c15c70 